\title{
On Building Evaluation System of Humanities and Social Sciences
}

\author{
Qian Sun $^{1} \&$ Hongwei Sun ${ }^{2}$ \\ ${ }^{1}$ Department of Social Science, Northwest University for Nationalities, Lanzhou, China \\ ${ }^{2}$ School of Environmental and Municipal Engineering, Lanzhou Jiaotong University, Lanzhou, China \\ Correspondence: Qian Sun, Department of Social Science, Northwest University for Nationalities, Lanzhou 730030, \\ Gansu, China. E-mail: esqian@126.com
}

Received: December 24, 2013

Accepted: January 19, 2014

Online Published: March 5, 2014

doi:10.5430/ijba.v5n2p107

URL: http://dx.doi.org/10.5430/ijba.v5n2p107

This work was supported by the Fundamental Research Funds for the Central Universities of Northwest University for Nationalities (Grant No.31920130129).

\begin{abstract}
In recent years, there are a lot of research on humanities and social science, but these researches are more general and substantial, less innovative. This mainly due to the academic evaluation system is not perfect. Therefore, to build a scientific and reasonable evaluation system of humanities and social sciences have become the common demand of academia. Based on the analysis of the necessity of the humanities and social science achievement evaluation system, this paper expounds the shortages of humanities and social science evaluation mechanism and its institutional guarantee.
\end{abstract}

Keywords: humanities and social science, evaluation system, institutional guarantee

\section{Introduction}

As humanities and social science research increase rapidly in China, academic evaluation has become a hot topic in academia and humanities and social science research. It is the common demand of academia to build a scientific and reasonable evaluation system.

At present, the humanities and social science researchers had made some beneficial exploration on humanities and social science evaluation, but overall there are still some problems, mainly include evaluation subject is not clear, evaluation purpose is not clear, evaluation standard is fuzzy, evaluation method is not diverse enough,evaluation system is not sound, etc., to some extent, these factors restricted the components of the humanities and social science evaluation system. Therefore, clearing evaluation purpose and evaluation direction is inevitable in the development of humanities and social science evaluation, and constructing scientific and reasonable evaluation system can also offer the base of ideas and methodology for the evaluation of practice.

\section{The Necessity of the Construction of Humanities and Social Science Evaluation System}

Academic evaluation of the first and most basic purpose is to promote the communication of valuable academic research results, and gradually become the important way to confirm researchers' academic achievement. On this base, to accelerate the standardization of the academic research appears as a kind of knowledge development in the higher stage of consciousness.

In recent years, with more and more researches in this area have been conducted in recent years, but in these research results, there are still less substantial and innovative achievements, in which there are quite a number of academic misconduct and corruption. It is generally believed that the fundamental reason is due to the lacking of a scientific and reasonable academic evaluation system, thus, to build a perfect evaluation system of humanities and social science has become an urgent and inevitable requirement for the development of social science and humanity.

\subsection{Modern University System Requires for Academic Autonomy and Freedom}

Academic autonomy and academic freedom are the foundation of modern university system. With the development of the modern university system, the higher requirements for academic research are put forward, academic evaluation is a king ring for effectively achieving academic autonomy and freedom. The academic evaluation system cannot 
guarantee the academic freedom and innovation, which will lead to disorderly competition, and runs in the opposite direction with the establishment of the modern university system. Therefore, in the criticism of quantitative evaluation, we need to think more about how to set up an academic evaluation system which should be taken by quality as the main standard while appropriating to lower the standard of quantitative assessment. Meanwhile, rewarding those who do have the research achievements of academic value and influence, in this way the innovation of the academic environment, the development of academic freedom and academic autonomy could be promoted.

\subsection{Requirements for a Scientific and Reasonable Evaluation System}

At present, Humanities and social science evaluation research in China is still in the exploration stage, healthy and orderly development of humanities and social science research need to be done by evaluation activity. Humanities and social science research has characteristics of complexity, diversity and performance in refinement of the subject, intersection and integration of different disciplines, which lead to the diversified final results. Single evaluation criterion cannot reasonably reflect the results of the humanities and social science, and the evaluation also involves many unquantifiable evaluation index, therefore, interdisciplinary, multidisciplinary and interdisciplinary evaluation requires the combination of quantitative and qualitative evaluation criteria. Deeply analyzing the complexity and diversity of humanities and social science and building perfect evaluation system is an inevitable requirement of humanities and social science research development.

\subsection{Humanities and Social Science Evaluation Depends on Scientific and Standard Evaluation System}

A full range of humanities and social science evaluation include three levels which are macro, meso and micro. Macro level refers to the evaluation of discipline, medium level refers to the evaluation of scientific research institutions, micro-level refers to the evaluation for scientific research achievements and scientific research personnel. Humanities and social science research evaluation mainly refers to the evaluation of the micro level, for the assessment of scientific research achievements related to personnel, project evaluation, evaluation of academic achievements, and many other issues. The choice of evaluation subject, evaluation method and evaluation standard needs a scientific and standard humanities and social science evaluation system, otherwise the further development of humanities and social science research will be limited.

\subsection{The Existing Rich Natural Science Research Evaluation Theory for Reference}

The current humanities and social science evaluation research in China is still at the exploratory stage, compared with natural science, humanities and social science research has some different characteristics, such as strong subjectivity, lag of benefits and value of collateral, the complexity of the evaluation objects, some uncertainty of the object of study, etc. Therefore, a more unique humanities and social science evaluation system should be built, which is different from natural science evaluation system. Natural science evaluation system started earlier than humanities, which formed some evaluation methods through practice has proved to be more mature, although there are many imperfections, accumulated some experience for reference.

\section{The Shortages of Humanities and Social Science Evaluation Mechanism}

In recent years, many scholars has carried on lots of positive exploration and research on the humanities and social science evaluation mechanism, however, so far, there is not a scientific and reasonable evaluation system. Overall, the current shortcomings in the Chinese humanities and social science evaluation mechanism mainly include:

\subsection{The Imperfection of the Evaluation Procedure Could Not Guarantee the Democracy and Impartiality of the} Evaluation

The importance of the evaluation procedures have been highly recognized for academia, so fair and reasonable evaluation procedures are called for establishing.

Currently, there is no scientific research management department or scientific researchers have put forward an evaluation program which can get everyone's approval and widespread application, the evaluation procedure is still in the less perfect stage, and its direct consequence is no guarantee for the evaluation of the democracy and justice.

Evaluation procedure is imperfect, which easily lead to the tendency of attaching great importance to the number and looking down on quality.To a certain extent, it contributed to the impetuous grandiose style of study and speculation. Evaluation program is not perfect which lead to "bureaucratic" and "specialization" gradually, so that part of the scientific research management department leadership and expert evaluation (awards) has increasingly become a "professional".

Part of the humanities and social science evaluation exists the phenomenon of "review expert"; Evaluation procedure is imperfect, disadvantageous academic masters, institutional leadership of idolatry and blindly follow, "celebrity 
effect" and "centralized around the evaluation results, lacking of democratic atmosphere, killed with different academic schools and academic views of experts and scholars say, the impartiality of the impact assessment.

3.2 Evaluation Method Is Difficult to Achieve Ideal Effect, the Interference of Non-Scientific Factors Cannot Be Ruled Out

Some more scientific, impartial method were derived in the current evaluation of humanities and social, comparing with peer review method and measurement evaluation method, such as communications, evaluation method, evaluation meeting, double-blind method, quantitative analysis, citation analysis and so on. Despite the design of these methods are very perfect on the "theory", but in the actual execution it may go out of form. Practitioners '(evaluation experts) personal preference make effect of the scientific method distorted, which often happen in practice. In the evaluation of humanities and social science, and evaluators' subjective bias is a lack of restrictions, which would cause injustice of the evaluation results. Therefore, the evaluation method will bring the congenitally deficiency the interference of non-scientific factors cannot be ruled out. Non-scientific factors mainly include: the relationship between human feelings, academic exchange and balance law, celebrity, left-leaning thoughts and level, index distribution, etc.

\subsection{The Supervision System Does Not Really Work, Which Cannot Regulate the Behavior of Evaluation Participants}

To establish a perfect supervision system is inherent in the humanities and social science evaluation. More and more supervision system and supervision method is put forward and implemented, but did not really work, to a certain extent, unable to regulate the behavior of evaluation of participants.

First, the evaluators' behavior is not standard. Due to the absence of supervision mechanism, evaluation of their own duties and responsibilities is not clear and it is hard to stick to their true opinions.

Second, due to a lack of responsibility and rewards and punishment mechanism, there is a slander or flatter phenomenon.

Third, the evaluation behaviors of organizers have not standardized. Evaluation organizers should be impartial third party, if supervision does not reach the designated position. All these non-standard behavior will affect the objectivity and impartiality of the humanities and social science evaluation.

\section{Construction of Humanities and Social Science Evaluation System}

Construction of humanities and social science evaluation system is the objective demand of the prosperous development of humanities and social science, but also can avoid frequent academic misconduct and inhibition of the academic corruption. There are many problems in academic evaluation process, such as academic dishonesty phenomenon, lacking of independent academic evaluation mechanism, the interference of non-academic factors and permeability, etc. All of these factors on the implementation of the humanities and social science evaluation caused serious interference, which affected the process of evaluation and its results, so the institutional defects need to be realized by institutional change.

\subsection{Establish Special Evaluation Institutions}

The development of humanities and social science research needs a scientific and reasonable evaluation system, and scientific evaluation organization is a guarantee to build evaluation system. At present, there are some evaluation agencies and organizations of humanities and social science research in China attaché to the government agencies or other entities units, but its role is not obvious, this is mainly due to the problems arising from the academic evaluation could not be certified and identified scientifically and effectively. It is inevitable to establish a special independent evaluation agency for the development of humanities and social science research.

Some scholars put forward that a social science evaluation center should be set up in China in the field of humanities and social science, and it should be a separate, dedicated and authoritative institutions, it can evaluate on academic research achievements, research projects, scientific research institutions, academic researchers and some of the other's assessment of the business which entrusted by academic institutions, only in this way the impartiality of the evaluation activity could be guaranteed.

Therefore, establishing special humanities and social science evaluation mechanism is not only the key way to realize the scientific, standardized development of humanities and social science evaluation, but also the realistic need for the prosperity of the humanities and social science development.

\subsection{Improving the System of Peer Review}

Humanities and social science research has the characteristics of vagueness, descriptive, so its not like a natural 
scientific results which could be repeatedly verified through scientific experiments and precise mathematical calculation, also cannot be measured by indicators and data.

Therefore, to determine the value of the research results by the experts engaged in the same or similar field, which has become the main evaluation methods in humanities and social science research, namely is peer review method. Peer review method is a kind of qualitative evaluation method, its characteristic is easy to be operated and used.

Due to the knowledge, research and analysis ability of peer experts, it is hard to avoid the inherent limitations of evaluation itself. However, the peer review method still has some random factors, such as the effects of valuator's subjective consciousness; limitations of peer experts' knowledge and experience, etc., which often make a biased result.

Especially the constantly produce of emerging, comprehensive, interdisciplinary subjects, scope expanding of the research, for those who applied strong social scientific research achievement, which affect achievement value standard should not only consider its academic value but also consider the results of economic value and social benefits.

At the same time, peer-review system should carry out expert avoidance principle, which focuses on effective restriction for peer experts, thus can avoid the interference of some non-academic factors. It could make an open, fair result to accept the supervision of academic circle.

\subsection{Actively Promote the "Representative Academic Achievements" System}

Representative academic achievements refer to those study results which could reflect academic level. Representative academic achievements were put into the practice initially in Tsinghua University, now it is already widely recognized in academic circles in China. It has a certain practical significance and has become an important evaluation index of humanities and social science research.

Academic papers and representative academic works are affirmed as the main output form by Tsinghua University, "one representative academic works" and "five academic articles" are identified as its representative achievements of the scientific research evaluation in the liberal arts. It not only embodies the negation of the number of academic achievements, but also embodies the pursuit of quality, the more is to effectively resist various administrative measures on evaluation mechanism of excessive intervention, so as to realize the organic combination of qualitative evaluation with quantitative evaluation. In addition, due to a long period for the publication of representative academic achievements, Tsinghua University put forward that the representative academic achievement should not be restricted by the evaluation cycle, which allows researchers to study carefully, rather than pursuit the number of achievements.

\section{Conclusion}

Based on the analysis of the necessity of the humanities and social science achievement evaluation system, a scientific and reasonable evaluation system of humanities and social sciences is built. This paper expounds the shortages of humanities and social science evaluation mechanism and its institutional guarantee.

\section{References}

Cheng, Ping. (2009). the academic evaluation system of humanities and social sciences. Journal of Chongqing University, 4, 71-76.

Liu, Dachun. (2009). A Survey of Evaluation of Chinese Humanities and Social Sciences. Journal of Chongqing University, 1, 45-50.

Qiu, Junping. (2002, March). SSCI: Significance, Characteristics and Amelioration. Wuhan University Journal (Social Sciences), 55(2), 232-236.

Ye, Jiyuan. (2010). Approaching Evaluation System in Humanities and Social Sciences. Journal of Nanjing University, 1, 51-55.

Zhu, Shaoqiang. (2007). The characteristics of humanities and social science research and impacts on research evaluation. Journal of Chongqing University (Social Science Edition), 5. 